\title{
Searches for Heavy Resonances with CMS
}

\author{
Christine McLean* \\ On Behalf of the CMS Collaboration, \\ Department of Physics \\ University of California, Davis, CA 95616, U.S.A \\ E-mail: ch.mcleandeern.ch
}

\begin{abstract}
We present a set of searches for new heavy resonances with the CMS detector at the LHC. Protonproton collision data recorded at a center-of-mass energy of $13 \mathrm{TeV}$ is used. Specifically, we consider searches for a $W^{\prime}$ boson decaying to a lepton and a neutrino, a $Z^{\prime}$ boson decaying to a pair of taus, and a heavy particle ( $Z^{\prime}$ boson or Randall Sundrum Kaluza Klein gluon) decaying to a pair of top quarks. The searches are performed by measuring the mass distributions of the final state particles and testing for deviations from the expected Standard Model background. In the high mass ranges accessible by the Run II LHC, the final state particles are expected to be produced with high transverse momenta. This leads to dijet event topologies, and the latest reconstruction techniques are employed to separate these signatures from backgrounds. The results are presented in terms of upper limits on the model cross sections.
\end{abstract}

Fourth Annual Large Hadron Collider Physics

13-18 June 2016

Lund, Sweden

${ }^{*}$ Speaker. 


\section{Introduction}

Many "Beyond-the-Standard-Model" (BSM) theories, such as little Higgs theories and models of extra dimensions, propose new resonances as an extension to the Standard Model (SM) of particle physics $[1,2,3,4]$. Recently, excesses observed at the LHC in the diphoton and diboson invariant mass spectra at $750 \mathrm{GeV}$ and $2 \mathrm{TeV}$ have provided motivation for resonance searches in the high mass regime $[5,6,7]$. This note focuses on searches for heavy bosons called $W^{\prime}$ and $Z^{\prime}$, as well as Randall-Sundrum Kaluza-Klein (RS KK) gluons. Section 2 examines the search for a heavy W-like boson that decays into a lepton and missing transverse energy $\left(\mathrm{E}_{\mathrm{T}}^{\text {miss }}\right)[8,9]$. Sections 3 and 4 explore searches for heavy Z-like bosons that couple preferentially to third generation particles, specifically taus and top quarks, respectively [10]. Section 4 also considers a model of RS KK gluons decaying into top pairs [11, 12]. All searches use data recorded by the Compact Muon Solenoid (CMS) experiment at the Large Hadron Collider (LHC) during the 2015 proton-proton run, with a collision energy of $\sqrt{s}=13 \mathrm{TeV}$ [13]. These analyses serve as updates to corresponding $8 \mathrm{TeV}$ searches. Due to the increase in center-of-mass energy, they utilize the latest identification techniques, such as jet substructure tools, to reconstruct the high energy event topologies.

\section{2. $W^{\prime} \rightarrow$ lepton $+\mathbf{E}_{\mathrm{T}}^{\text {miss }}$}

In BSM theories, the charged heavy gauge boson is usually referred to as the $W^{\prime}$. This note presents searches for a $W^{\prime}$ decaying into a charged lepton (electron, muon, or hadronic tau) and an invisible particle (neutrino, dark matter) ([8,9]). The electron/muon (tau) analysis uses $2.2(2.3)$ $\mathrm{fb}^{-1}$ of $13 \mathrm{TeV}$ CMS data. Figure 1 illustrates the hadronic tau $\left(\tau_{h}\right)$ decay channel of the $W^{\prime}$.

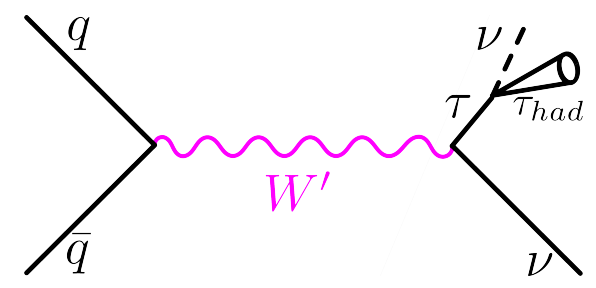

Figure 1: Illustration of the $W^{\prime} \rightarrow \tau v$ decay channel, in which the tau decays hadronically.

Due to its high mass, the $W^{\prime}$ is produced almost at rest and decays into particles with high energies. Therefore, the analysis looks for events with an isolated lepton with high transverse momentum $\left(p_{\mathrm{T}}\right)$ and large missing transverse energy $\left(E_{\mathrm{T}}^{\text {miss }}\right)$, which indicates the presence of a neutrino. The main irreducible background comes from off-shell SM $W \rightarrow l v$ production, with $l=e, \mu, \tau$. This background is estimated from Monte Carlo (MC) simulation, as are the smaller contributions from Drell-Yan, $t \bar{t}$, single top, and diboson processes. The QCD multijet background is estimated from data, but is mostly rejected by a cut on $p_{\mathrm{T}} / E_{\mathrm{T}}^{\text {miss }}$. In order to select for $W^{\prime} \rightarrow l v$ events and suppress background, the following kinematic cuts are applied:

- The difference in the azimuthal angle between the lepton $p_{\mathrm{T}}$ and the missing transverse momentum is required to be $\left|\Delta \phi\left(\vec{p}_{\mathrm{T}}^{l}, \vec{p}_{\mathrm{T}}^{\text {miss }}\right)\right|>2.5$ (2.4) for the electron/muon (tau) channel. This ensures a back-to-back event topology. 
- The ratio of lepton $p_{\mathrm{T}}$ and missing transverse energy must be $0.4(0.7)<p_{\mathrm{T}} / E_{\mathrm{T}}^{\text {miss }}<1.5$ (1.3) for the electron/muon (tau) channel.

Figure 2 illustrates how these two requirements provide good signal to background discrimination after event selection in the tau channel. Additionally, one can see that the data generally agrees well with the estimated background.
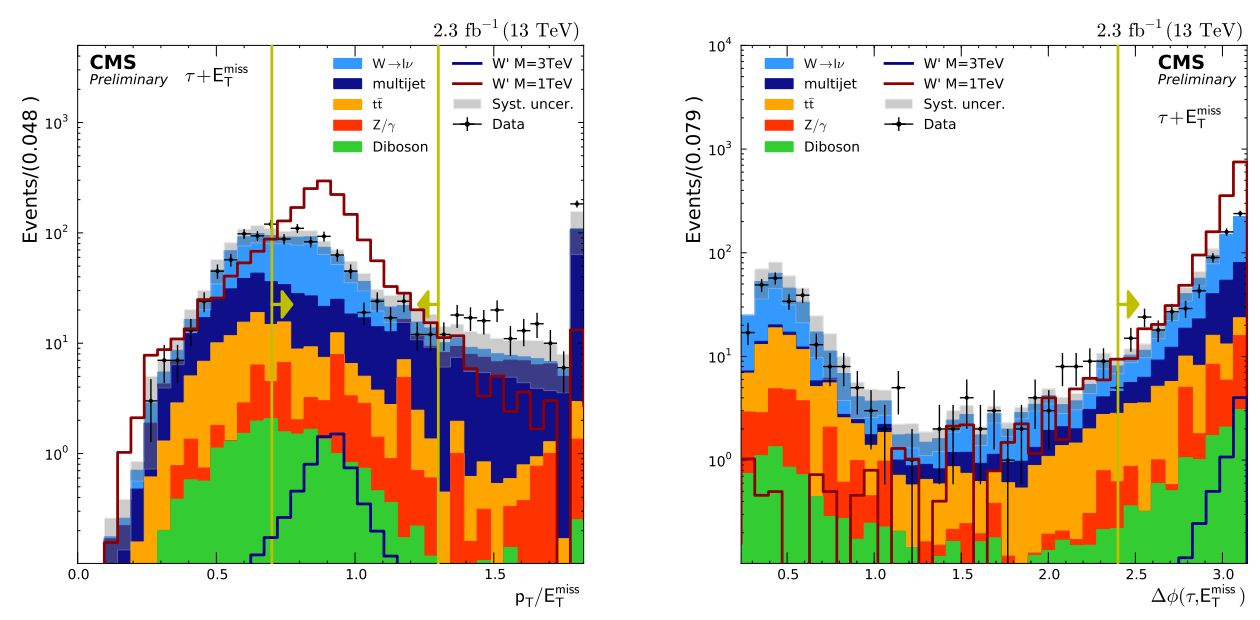

Figure 2: Distributions of $W^{\prime}$ kinematic variables after the initial selection. The left (right) plot shows the distribution of $p_{\mathrm{T}} / E_{\mathrm{T}}^{\text {miss }}\left(\Delta \phi\left(p_{\mathrm{T}}^{l}, E_{\mathrm{T}}^{\text {miss }}\right)\right)$ for the $\tau_{h}$ decay channel. The solid histogram shows the background, the blue and brown curves show the expected signal, the black points show the data, and the vertical lines indicate the signal selection.

The sensitive variable used in the $W^{\prime}$ searches is the transverse invariant mass, $\mathrm{M}_{\mathrm{T}}$, which is defined as $\mathrm{M}_{\mathrm{T}}=\sqrt{2 p_{\mathrm{T}}^{l} E_{\mathrm{T}}^{\text {miss }}\left(1-\cos \left[\Delta \phi\left(\vec{p}_{\mathrm{T}}^{l}, \vec{p}_{\mathrm{T}}^{\text {miss }}\right)\right]\right)}$, where $E_{\mathrm{T}}^{\text {miss }}=\left|\vec{p}_{\mathrm{T}}^{\text {miss }}\right|$. The analysis looks for a $W^{\prime}$ signal peaking over a falling $\mathrm{M}_{\mathrm{T}}$ background spectrum. Figure 3 shows the $\mathrm{M}_{\mathrm{T}}$ distributions for all three decay channels after the full event selection. Again, good data/background agreement is seen and no signs of new physics are observed.

Upper limits are set on the production cross section times branching ratio of a $W^{\prime}$ boson with a mass above $1 \mathrm{TeV}$. Figure 4 shows the 95\% Confidence Level (CL) expected and observed limits for the combined muon and electron analysis, as well as the hadronic tau analysis. The observed limit excludes $W^{\prime}$ masses below 4.4 (3.3) TeV for the electron/muon (tau) channel. This represents a significant improvement over the $8 \mathrm{TeV}$ results, which only excluded masses up to 3.3 (2.7) $\mathrm{TeV}$ in the electron/muon (tau) channel. 

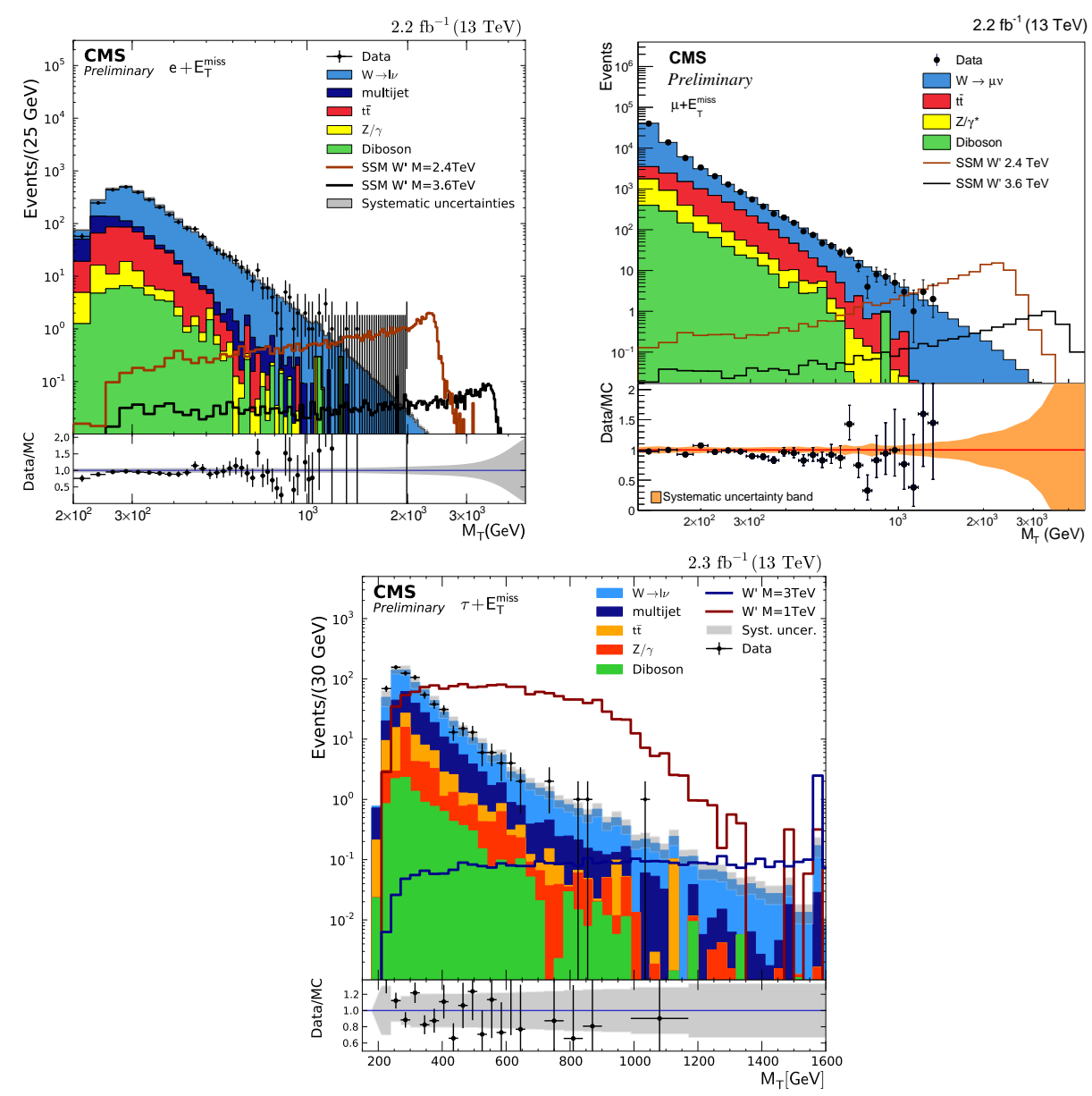

Figure 3: Distributions of $\mathrm{M}_{\mathrm{T}}$ after the event selection for the electron (top left), muon (top right), and tau (bottom) decay channels. Although good signal to background discrimination can be seen for this variable, no excess is observed.
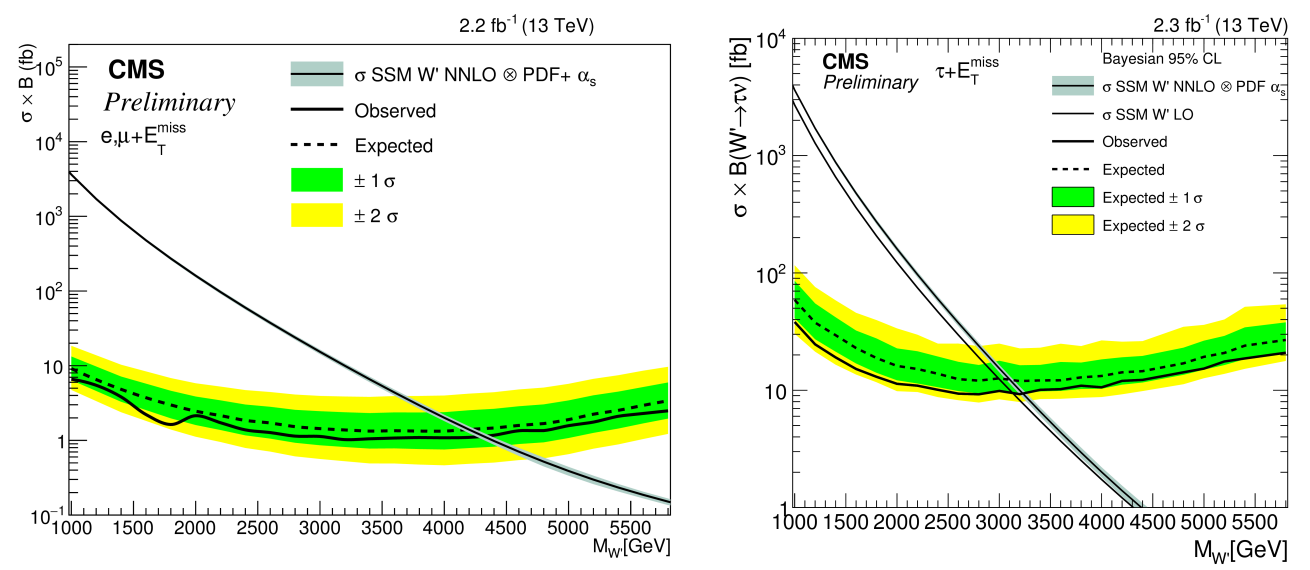

Figure 4: Upper limits on the cross section times branching ratio of the $W^{\prime}$ boson as a function of mass. The electron/muon (tau) channel limits are shown in the left (right) plot. The dashed line shows the median expected limits while the solid line gives the observed limit. The green (yellow) band gives the one (two) sigma expected limit band. The theoretical cross section prediction is also shown. 


\section{3. $Z^{\prime} \rightarrow \tau \tau$}

Another heavy gauge boson predicted by BSM theories is the neutral $Z^{\prime}$, which in some theories couples preferentially to third generation fermions. Here we consider a $Z^{\prime}$ that solely decays to pairs of tau leptons, using $2.2 \mathrm{fb}^{-1}$ of $13 \mathrm{TeV}$ CMS data [10]. Four decay channels are considered: $Z^{\prime} \rightarrow \tau_{e} \tau_{\mu}, \tau_{e} \tau_{h}, \tau_{\mu} \tau_{h}$, or $\tau_{h} \tau_{h}$, where $\tau_{e}\left(\tau_{\mu}\right)$ indicates a tau decaying to an electron (muon) plus a neutrino and $\tau_{h}$ indicates a tau that decays hadronically, as illustrated in the previous section. The analysis is characterized by a search for events with a high $p_{T}$ isolated lepton and $E_{\mathrm{T}}^{\text {miss }}>30 \mathrm{GeV}$.

As with the previous analysis, the high mass of the $Z^{\prime}$ leads to a back-to-back event topology. The corresponding kinematics are utilized to distinguish signal from background:

- An isolated electron or muon, in the case of the $\tau_{e}$ and $\tau_{\mu}$ decays, or an isolated $\tau_{h}$.

- An angular distribution described by $\cos \Delta \phi\left(\tau_{1} \tau_{2}\right)<-0.95$.

- A geometrical cut is made on the momentum in the direction of the missing transverse energy such that $\xi=p_{\xi}-3.1 \times p_{\xi}^{v i s}>-50 \mathrm{GeV}$. The $\hat{\xi}$ direction is defined by the unit vector in the direction bisecting the visible $\tau$ lepton decay products in the plane transverse to the direction of the beam. The variables contributing to $\xi$ are defined as $p_{\xi}^{v i s}=\left(\vec{p}_{T}^{\tau_{1}}+\vec{p}_{T}^{\tau_{2}}\right) \cdot \hat{\xi}$ and $p_{\xi}=\left(\vec{p}_{T}^{\tau_{1}}+\vec{p}_{T}^{\tau_{2}}+\vec{E}_{T}^{m i s s}\right) \cdot \hat{\xi}$.

The QCD, W+jets, and Drell-Yan+jets backgrounds are estimated from data. The SM $t \bar{t}$ background is estimated from MC simulation, and its contribution is minimized by requiring events with no b-tagged jets.

The sensitive variable used in this search is the $Z^{\prime}$ mass, reconstructed using the missing transverse energy and the visible $\tau$ decay products:

$$
m\left(\tau_{1}, \tau_{2}, E_{T}^{\text {miss }}\right)=\sqrt{\left(E_{\tau_{1}}+E_{\tau_{2}}+E_{T}^{\text {miss }}\right)^{2}-\left(\vec{p}_{\tau_{1}}+\vec{p}_{\tau_{2}}+\vec{E}_{T}^{m i s s}\right)^{2}}
$$

The analysis looks for a broad $Z^{\prime}$ signal peaking over a falling background spectrum. Figure 5 shows the mass distribution for the $\tau_{\mu} \tau_{h}$ channel. After the event selection, this variable shows very good signal/background discrimination, though no excess can be seen in the data. Limits are set as a function of $Z^{\prime}$ mass, combining all four decay channels, as can be seen in Figure 6. The mass of the $Z^{\prime}$ is excluded up to $2.1 \mathrm{TeV}$, an improvement upon the $8 \mathrm{TeV}$ limits of $1.3 \mathrm{TeV}$. This analysis is the first $13 \mathrm{TeV}$ exotic $\tau \tau$ result. 


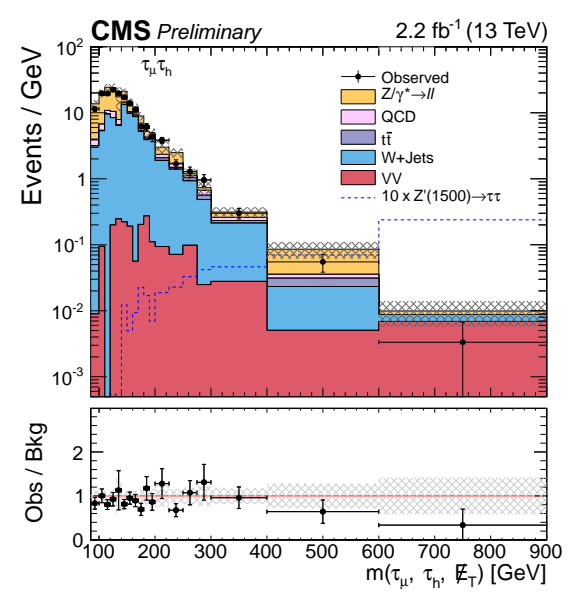

Figure 5: Distributions of the Z' mass, reconstructed using the $\tau_{\mu} \tau_{h}$ channel. The solid histogram shows the background, the blue dotted curve shows the expected signal, and the black points show the data. Although good signal to background discrimination is seen, no excess is observed.

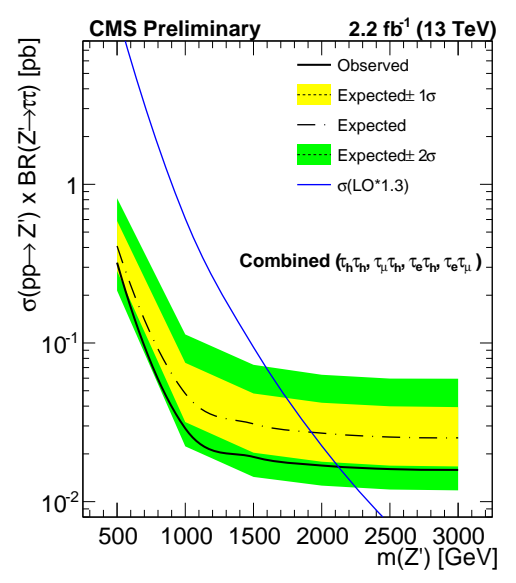

Figure 6: Combined upper limits on the cross section times branching ratio of the $Z^{\prime}$ boson as a function of mass. The dashed line shows the median expected limits while the solid line gives the observed limit. The one (two) sigma expected limit band is shown in green (yellow). The solid blue line gives the theoretical cross section prediction.

\section{Top Pair Resonances}

Next, we present a pair of searches for heavy resonances coupling preferentially to top quarks, using $2.6 \mathrm{fb}^{-1}$ of $13 \mathrm{TeV}$ CMS data. Models of a leptophobic topcolor $Z^{\prime}$ - whose width is either $1 \%, 10 \%$, or $30 \%$ of its mass - and an RS KK gluon are considered [11, 12]. The searches focus on two final states: the all hadronic final state, in which both top quarks decay hadronically, and the semileptonic final state, in which one top quark decays hadronically and the other decays leptonically. In both cases, due to the high mass of the $Z^{\prime}$, the events are described by a dijet topology in which each top is boosted and its decay products are merged into a single jet. To identify the hadronically-decaying top quarks and distinguish them from SM backgrounds, we use the newly 


\begin{tabular}{lcc} 
Event category & $|\Delta y|$ & Number of AK8 jets with at least one subjet b-tag \\
\hline A & $<1.0$ & 0 \\
B & $<1.0$ & 1 \\
C & $<1.0$ & 2 \\
D & $>1.0$ & 0 \\
E & $>1.0$ & 1 \\
F & $>1.0$ & 2
\end{tabular}

Table 1: Definition of event categories for the all-hadronic channel. Here, $|\Delta y|$ is the difference between the rapidities of the two top-tagged jets.

developed CMS Top Tagger Version 2 (CMSTTv2) algorithm [14]. It uses jet substructure tools to remove excess radiation from jets and properly determine their masses and the number of subjets they contain. We also make use of the Combined Secondary Vertex v2 (CSVv2) algorithm to indentify the $b$ quarks resulting from the top decays $[15,16]$.

The dijet event selection requires two back-to-back high $p_{T}$ jets, in the case of the all hadronic decay mode. The semileptonic selection requires an energetic lepton (electron or muon), missing energy, a large-radius high $p_{T}$ jet, and a smaller radius jet. In order to increase sensitivity, each analysis separates its events into six categories. The all-hadronic channel requires two top-tagged jets that are separated into event categories based on the rapidity (y) separation between the two jets and the number of top jets with subjet b-tags (Table 1). The semileptonic selection separates events into electron and muon channels. Then, each channel is separated into events with either 1 top-tag, 0 top-tags and $1 \mathrm{~b}$-tagged jet, or 0 top-tags and $0 \mathrm{~b}$-tags.

For the all-hadronic channel, the SM backgrounds consist of $t \bar{t}$, which is estimated from simulation, and non-top multijet (NTMJ), which is estimated from data. The semileptonic SM background consists of $t \bar{t}, \mathrm{~W}+$ jets, single top, Drell-Yan + jets, VV, and NTMJ/QCD, all of which are estimated from simulation. The kinematics of the boosted event topology are utilized to determine additional selection criteria to remove background from the semileptonic channel. In a lower energy regime, an isolation requirement would be placed on the lepton, but in the boosted regime the lepton can overlap with the b-jet. Therefore, a $2 \mathrm{D}$ requirement is made: $\Delta R(l, j)>0.4$ or $p_{T}^{\text {rel }}(l, j)$ $>20 \mathrm{GeV}$, where $j$ indicates the small-radius jet with minimum angular separation to the lepton (electron or muon) 1 , and $p_{T}^{\text {rel }}(l, j)$ is defined as the magnitude of the lepton momentum orthogonal to the axis of jet $j$. The left plot in Fig. 7 shows the high QCD background rejection power of the $2 \mathrm{D}$ cut, as compared to a simple isolation requirement. Additionally, a $\chi^{2}<30$ cut is made, where $\chi^{2}$ is defined as

$$
\chi^{2}=\left(\frac{M_{l e p}-\bar{M}_{l e p}}{\sigma_{M_{l e p}}}\right)^{2}+\left(\frac{M_{\text {had }}-\bar{M}_{\text {had }}}{\sigma_{M_{\text {had }}}}\right)^{2},
$$

where $M_{l e p}$ and $M_{\text {had }}$ are the invariant masses of the reconstructed leptonic and hadronic top quark, respectively. The right plot in figure 7 shows the distribution of the $\chi^{2}$ variable.

Top-tagging is done using CMSTTv2 - the latest top-tagger developed for CMS in Run II of the LHC. It uses the softdrop grooming algorithm, which removes soft and collinear radiation from a jet, to determine the softdrop mass $\left(M_{\mathrm{SD}}\right)$ of the top jet [17]. It also places an N-subjettiness $\left(\tau_{32}\right)$ requirement on the jet. $\mathrm{N}$-subjettiness distinguishes 3-pronged jet substructure, which is 

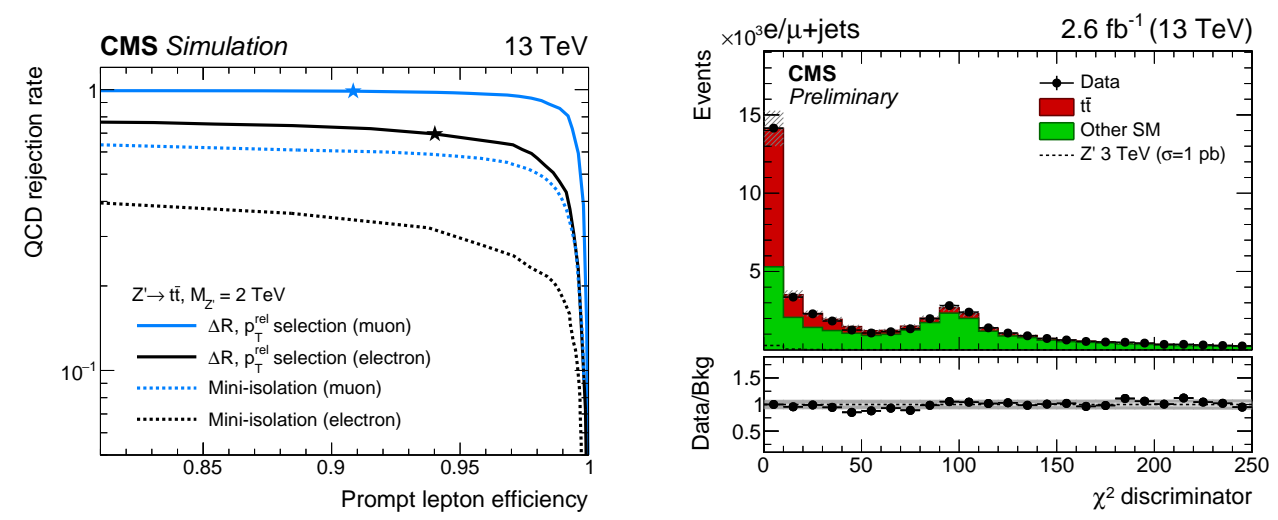

Figure 7: Kinematic variables used in the semileptonic event selection. Left: QCD rejection rate as a function of the reconstruction efficiency of prompt leptons from $\mathrm{W}$ decays in signal events. A comparison is made between the selections based on an isolation requirement (dashed curves) and a 2D cut (solid curves) for the muon (blue) and electron (black) channels. The stars indicate the working points used. A large gain in QCD rejection can be seen when the $2 \mathrm{D}$ cut is used instead of the isolation requirement. Right: The distribution of the $\chi^{2}$ variable, where the red histogram shows SM $t \bar{t}$, the green is other SM background, the dotted curve is the signal, and the black points are data. The $\chi^{2}<30$ cut keeps most of the $t \bar{t}$ events and rejects most of the other SM background.

indicative of a top jet, from non-top jets and is especially useful for distinguishing signal from NTMJ background [18]. The top-tagging working point requires the top jets to have $110 \mathrm{GeV}<$ $M_{\mathrm{SD}}<210 \mathrm{GeV}$ and $\tau_{32}<0.69$, which corresponds to a $3 \%$ NTMJ mistag rate in simulation. Figure 8 shows "N-1" plots for these two cuts after the semileptonic selection. A nice top peak can be seen in the softdrop mass distribution, and very good data/MC agreement is observed in both plots.
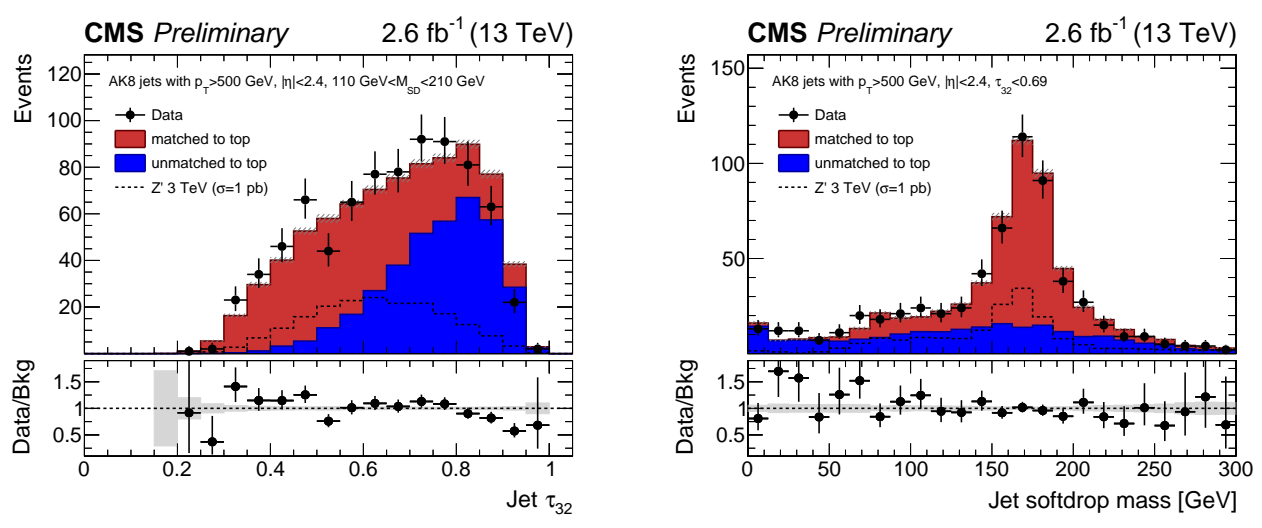

Figure 8: Distributions of the $\mathrm{N}$-subjettiness ratio $\tau_{32}$ (left) and the softdrop mass $M_{\mathrm{SD}}$ (right) in data and simulation after the semileptonic signal selection. The distribution of $\tau_{32}$ is shown after the selection $110<M_{\mathrm{SD}}<210 \mathrm{GeV}$ and the distribution of $M_{\mathrm{SD}}$ is shown after the selection on $\tau_{32}<0.69$.

The sensitive variable in this analysis is the $t \bar{t}$ invariant mass - we look for a signal peaking over the falling background spectrum. Figure 9 shows the mass distributions for the $|\Delta y|<1$, one subjet b-tag category in the all-hadronic channel and the combined $\mathrm{e} / \mu$, one top-tag category in the semileptonic channel. After the event selection, the invariant mass spectrum shows reasonable 
signal/background discrimination, though no excess can be seen in the data. Limits are set as a function of mass for the four signal hypotheses, combining all six event categories for both the allhadronic and semileptonic analyses. Figure 10 shows the limits set by both analyses for an extrawide $Z^{\prime}$, while Table 2 shows the observed limits for all four signal hypotheses. These results show some improvement in sensitivity at high mass, as compared with the $8 \mathrm{TeV}$ results. The sensitivity is expected to increase with the combination of the semileptonic and all-hadronic channels, as well as the addition of the 2016 data.
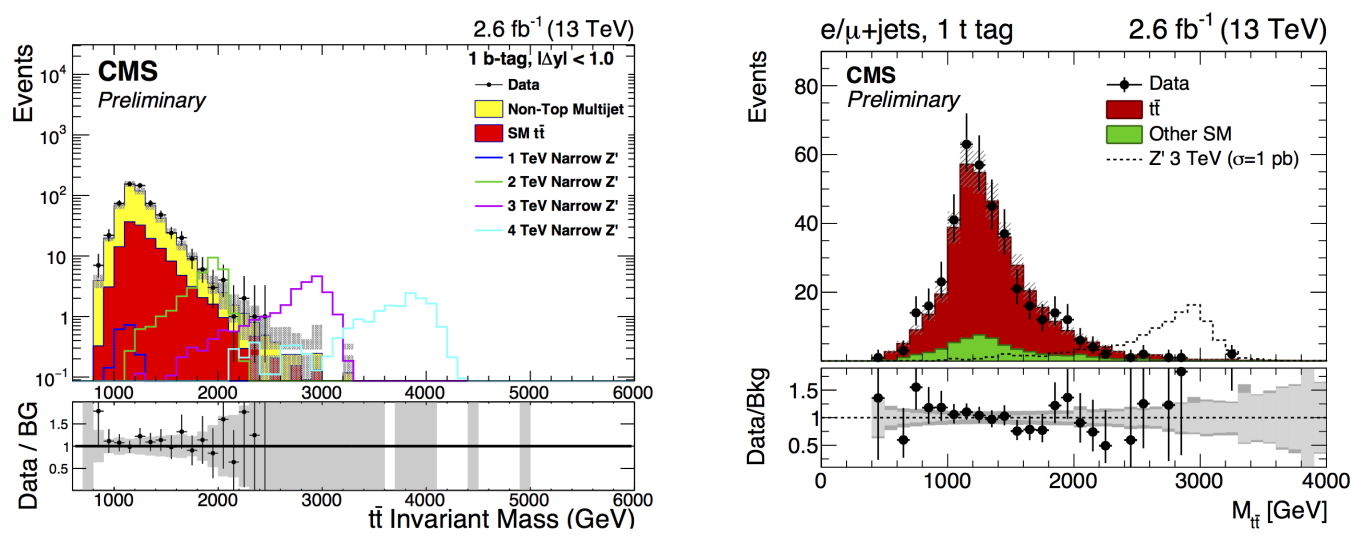

Figure 9: Distributions of the $t \bar{t}$ invariant mass after the Category B all-hadronic (left) and combined e/ $\mu$, one top-tag semileptonic (right) event selection. The solid histograms show the background, the curves show the expected signal, and the black points show the data. Although good signal to background discrimination is seen, no excess is observed.
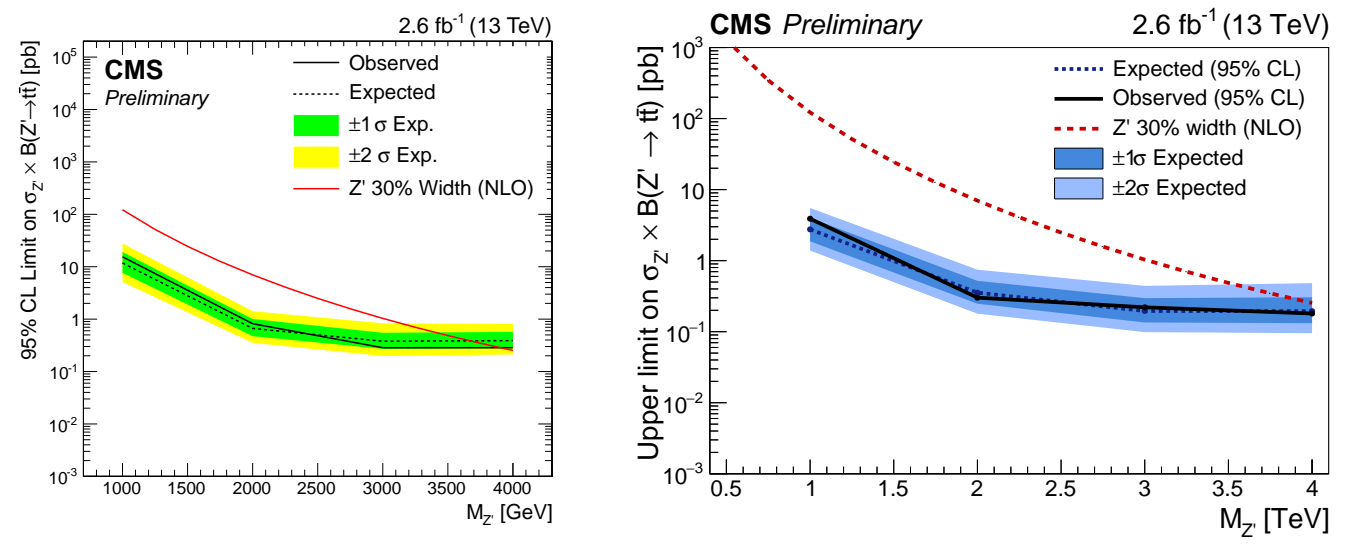

Figure 10: $95 \%$ CL limits on the cross section times branching ratio of the extra-wide $Z^{\prime}$ boson as a function of mass. The dashed black line shows the median expected limits, while the solid black line gives the observed limit. The one sigma expected limit band is shown in green (dark blue) for the all-hadronic (semileptonic) channel, while the two sigma band is shown in yellow (light blue). The red line gives the theoretical cross section predictions.

\section{Summary}

Many heavy resonance searches have been performed at CMS during Run II of the LHC. We 


\begin{tabular}{lcccc} 
& \multicolumn{4}{c}{ Observed Limit } \\
Decay Channel & Narrow Z' & Wide Z' & Extra Wide Z' & RS KK Gluon \\
\hline Semileptonic & $0.6-2.3$ & $0.5-3.4$ & $0.5-4.0$ & $0.5-2.9$ \\
All-Hadronic & $1.4-1.6$ & $1.0-3.3$ & $1.0-3.8$ & $1.0-2.4$
\end{tabular}

Table 2: Comparison of the observed mass exclusion results for the semileptonic and all-hadronic analyses.

explored searches for BSM $W^{\prime}$ and $Z^{\prime}$ bosons, as well as RS KK gluons, that were performed using $2.2-2.6 \mathrm{fb}^{-1}$ of $201513 \mathrm{TeV}$ proton-proton collision data. No signs of new physics have been observed. However, mass exclusion limits were set, which show significant improvement over the previous $8 \mathrm{TeV}$ results. The analyses will continue to improve in sensitivity as 2016 data is added.

\section{References}

[1] N. Arkani-Hamed, A. G. Cohen, and H. Georgi, Electroweak symmetry breaking from dimensional deconstruction, Phys. Lett. B $\mathbf{5 1 3}$ (2001) 232 [hep-ph/0105239].

[2] N. Arkani-Hamed, S. Dimopoulos, and G. R. Dvali, The hierarchy problem and new dimensions at a millimeter, Phys. Lett. B $\mathbf{4 2 9}$ (1998) 263 [hep-ph/9803315].

[3] L. Randall and R. Sundrum, A large mass hierarchy from a small extra dimension, Phys. Rev. Lett. 83 (1999) 3370 - 3373 [hep-ph/9905221].

[4] L. Randall and R. Sundrum, An alternative to compactification, Phys. Rev. Lett. 83 (1999) 4690 4693 [hep-th/9906064].

[5] A. Faraggi and M. Guzzi, Extra Z's and W's in heterotic-string derived models, Eur. Phys. J. 75 (2015) 537 [1507.07406].

[6] T. Li, J. Maxin, V. Mayes, and D. Nanopoulos Diboson Excesses in Leptophobic U(1) LP Models from String Theories Phys. Rev. D 93 (2016) 045007 [1509.06821].

[7] CMS Collaboration Search for new physics in high mass diphoton events in $\sqrt{s}=13 \mathrm{TeV}$, CMS-PAS-EXO-15-004.

[8] CMS Collaboration Search for SSM W production, in the lepton+MET final state at a center-of-mass energy of $13 \mathrm{TeV}$, CMS-PAS-EXO-15-006.

[9] CMS Collaboration Search for $W$ decaying to tau lepton and neutrino in proton-proton collisions at $\sqrt{s}=13 \mathrm{TeV}$, CMS-PAS-EXO-16-006.

[10] CMS Collaboration Search for new physics with high-mass tau lepton pairs in pp collisions at $\sqrt{s}=$ $13 \mathrm{TeV}$ with the CMS detector, CMS-PAS-EXO-16-008.

[11] CMS Collaboration Search for $t \bar{t}$ resonances in boosted semileptonic final states in pp collisions at $\sqrt{s}$ $=13 \mathrm{TeV}$, CMS-PAS-B2G-15-002.

[12] CMS Collaboration Search for top quark-antiquark resonances in the all-hadronic final state at $\sqrt{s}=$ $13 \mathrm{TeV}$, CMS-PAS-B2G-15-003.

[13] CMS Collaboration, The CMS Experiment at the CERN LHC, JINST 3 (2008) S08004.

[14] CMS Collaboration Top Tagging with New Approaches CMS-PAS-JME-15-002.

[15] CMS Collaboration Identification of b-quark jets with the CMS experiment, JINST 8 (2013) P04013 [1211.4462]. 
[16] CMS Collaboration Identification of b quark jets at the CMS Experiment in the LHC Run 2, CMS-PAS-BTV-15-001.

[17] A. J. Larkoski, S. Marzani, G. Soyez, and J. Thaler Soft Drop, JHEP 05 (2014) 146 [1402.2657].

[18] J. Thaler and K. Van Tilburg Identifying Boosted Objects with N-subjettiness, JHEP 1103 (2011) 015 [1011.2268]. 